\title{
多取代烯丙基硅化合物的立体选择性合成研究
}

\author{
姜铨何玲* 李卫东* \\ (重庆大学药学院 天然产物全合成与创新药物研究重庆市重点实验室 重庆 401331)
}

\begin{abstract}
摘要 烯丙基硅化合物是一类重要的有机合成中间体, 能参与多种类型的化学转化反应, 被广泛应用于 $\mathrm{C}-\mathrm{C}$ 键构建 和复杂化合物的合成反应中, 探索官能化烯丙基硅化合物的立体选择性合成对丰富有机合成方法学研究有重要意义. 利用有机硅取代的环丙基三级醇化合物的 Julia 烯烃化反应，得到了一系列新型的烷氧甲基取代的高碘代烯丙基硅化合 物. 相对于烷基取代的三级醇底物, 芳基取代的三级醇底物能得到立体选择性更好的产物.
\end{abstract}

关键词＼cjkstart烯丙基硅; Julia 烯烃化反应; 环丙基甲醇; 格氏试剂

\section{Studies on the Stereoselective Synthesis of Functionalized Allylsilane Compounds}

\author{
Jiang, Quan He, Ling* Li, Weidong* \\ ( ${ }^{a}$ Chongqing Key Laboratory of Natural Product Synthesis and Drug Research, School of Pharmaceutical Sciences, \\ Chongqing University, Chongqing 401331)
}

\begin{abstract}
Allylsilanes have emerged as synthetically useful intermediates that can undergo a variety of chemical transformations and have been found versatile applications in $\mathrm{C}-\mathrm{C}$ bond construction as well as in preparation of complex molecules. Exploration on the stereoselective route to functionalized allylsilanes is of great significance for the development of organic synthetic methodology. An efficient pathway to a series of novel alkoxy-substituted homoiodio-allylsilanes via the Julia olefination of silylmethyl cyclopropyl carbinols was described. Carbinols derived from cyclopropyl aryl ketone were evidenced to be superiorer in controlling the stereoselectivity of the products than the alkyl equivalents.
\end{abstract}

Keywords allylsilane; Julia olefination; cyclopropyl carbinol; Grignard reagent

烯丙基硅化合物是一种重要的有机合成子, 自 1948 年 Sommer 等 ${ }^{[1]}$ 发现其与亲电试剂的反应以来, 已 被广泛应用于 $\mathrm{C}-\mathrm{C}$ 键的构建 ${ }^{[2]}$ 和复杂天然产物的合成 研究 ${ }^{[3]}$ 中. 烯丙基硅化合物结构中同时存在硅原子和烯 丙基，弱极性的 $\mathrm{C}-\mathrm{Si}$ 键赋予了烯丙基硅化合物相较于 其它烯丙基金属试剂更好的稳定性，便于制备和储存; 同时，硅原子特殊的电子结构使得烯丙基硅化合物在化 学转化过程中能有更优异的表现, 比如硅原子能通过 $\alpha$ 效应或 $\beta$-效应稳定 $\alpha$-碳负离子、 $\alpha$-碳自由基、 $\beta$-碳正离 子和 $\beta$-碳自由基, 从而控制反应的选择性 ${ }^{[2 \mathrm{~d}]}$. 正是由于 烯丙基硅化合物的这些优势, 越来越多的合成方法被化 学家们发展 ${ }^{[4]}$ 并加以利用, 而光学纯烯丙基硅合成方法 的发展 ${ }^{[5]}$ 进一步推动了烯丙基硅化合物在不对称合成中 的应用 ${ }^{[6]}$. 烯丙基硅化合物的主要合成方法包括 Wittig
反应、取代硅烷的消除反应、1,3-二烯的 1,4-硅氢化反 应、烯烃与含硅金属试剂的偶联反应等, 但这些合成方 法往往不能很好地控制所得烯丙基硅化合物双键的构 型而得到混合物，极大地影响烯丙基硅在有机合成中的 应用. 因此, 发展高效高立体选择性的烯丙基硅合成方 法具有重要意义.

2004 年, 我们课题组 ${ }^{[7]}$ 发展了一种简洁高效的合成 碘代烯丙基硅的方法(Scheme 1), 利用带有大位阻基团 的硅甲基格氏试剂 $\mathrm{Me}_{2} \mathrm{PhSiCH}_{2} \mathrm{MgCl}$ 与环丙基酮的亲 核反应得到环丙基取代的 $\beta$-差基硅烷化合物，进而在 $\mathrm{MgI}_{2} \cdot\left(\mathrm{OEt}_{2}\right)_{2}$ 作用下选择性地发生 Julia 烯烃化反应，高 效合成了带有卤原子的烯丙基硅化合物，该类官能化的 烯丙基硅化合物在萜类天然产物全合成中应用成功 ${ }^{[8]}$. 该方法可应用于环状和链状碘代烯丙基硅化合物的合

* Corresponding authors. E-mail: heling2015@cqu.edu.cn; wdli@cqu.edu.cn Received April 30, 2019; revised July 25, 2019; published online August 30, 2019.

Project supported by the National Natural Science Foundation of China (Nos. 21672030, 21702023).

国家自然科学基金(Nos. 21672030, 21702023 )资助项目. 
成, 但合成链状烯丙基硅化合物双键的立体选择性往往 并不理想, 得到两种构型的混合产物 ${ }^{[7,8 b]}$ (Scheme 1). 因 此, 发展该类型官能化烯丙基硅化合物高效高立体选择 性合成方法对其在全合成中应用具有重要意义.
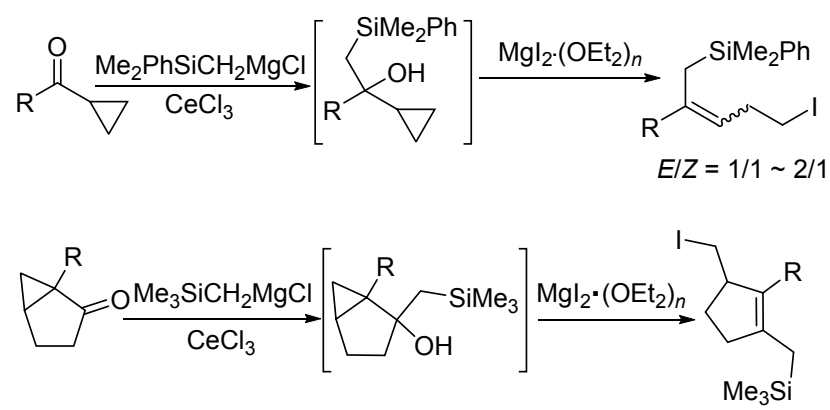

图式 1 基于 Julia 烯烃化反应合成碘代烯丙基化合物

Scheme 1 Synthesis of iodo-substituted allylsilanes based on the Julia olefination

2005 年, 我们课题组 ${ }^{[9]}$ 在利用 Julia 卤代烯烃化反应 合成多取代烯烃过程中, 发现环丙基甲醇化合物结构中 差基 $\beta$ 位带有乙酰氧基(OAc)的底物的卤化开环反应能 高立体选择性地得到 $E$ 式构型烯烃. 推测可能是反应过 程中形成的碳正离子中间体结构中, 酰基与碳正离子形 成了亚稳态五元环阳离子结构, 稳定了反应中间体, 同 时控制了后续碘离子进攻环丙环的方向, 得到单一构型 的产物(Scheme 2a). 基于以上反应机理推测, 本研究工 作通过在硅原子上引入能稳定碳正离子的取代基 $\left(\mathrm{OR}^{2}\right)$, 探索利用 $\beta$-环丙基- $\beta$-差基硅化合物的 Julia 烯烃化反应 立体选择性合成官能化烯丙基硅的可行性(Scheme 2b).

(a)<smiles>[R]C(O)(COC(C)=O)C1CC1</smiles>

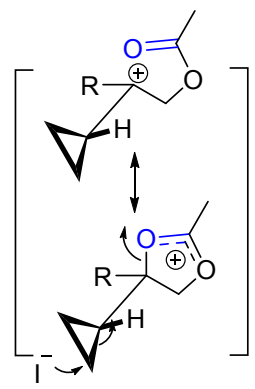

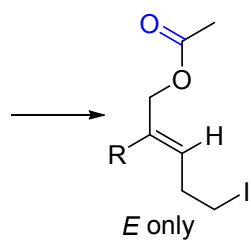

(b)<smiles>[R]OC[Si](C)(CC([R])(O)C1CC1)C1CC1</smiles><smiles>[R]OC[Si](C)(C)CC([R2])[C@H]1CC1C1CC1</smiles>

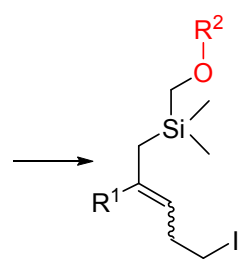

图式 2 官能化烯丙基硅化合物的立体选择性合成 Scheme 2 Stereoselective synthesis of functionalized allylsilane compounds

\section{1 结果与讨论}

烷氧基取代的 $\beta$-环丙基- $\beta$-羟基硅化合物利用相应 的有机硅格氏试剂与环丙基酮的亲核加成反应即可得 到. 首先探索了合成有机硅格氏试剂需要的烷氧甲基氯 甲基二甲基硅 1 的合成. $\alpha$-卤代甲基硅化合物的合成可 通过卤代卤甲基硅烷的亲核取代反应实现，常用的亲核 试剂是有机锂试剂和格氏试剂 ${ }^{[10]}$. 设计了利用甲氧基 甲基格氏试剂 $\mathbf{3}$ 与氯甲基二甲基氯硅烷的亲核反应合成 烷氧甲基取代的氯甲基硅烷化合物. 在 $-30{ }^{\circ} \mathrm{C}$ 条件 下 $^{[11]}$, 四氢呋喃(THF)作为溶剂，甲氧基甲基格氏试剂 与氯甲基二甲基氯硅烷能顺利发生反应，以 $45.2 \%$ 的产 率得到氯甲基-甲氧基甲基-二甲基硅烷(1) (Scheme 3).

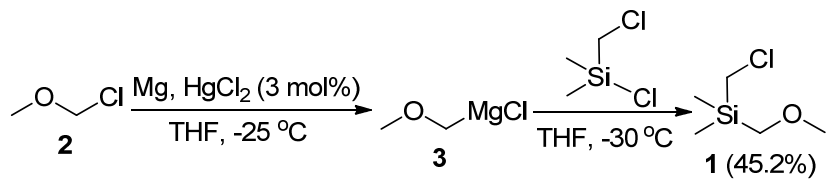

图式 3 化合物 1 的合成

Scheme 3 Synthesis of compound 1

接下来，考察带有烷氧基甲基烯丙基硅的合成条 件. 参考本课题组 ${ }^{[7]}$ 以往关于烯丙基硅化合物的合成条 件，以 $\mathrm{CeCl}_{3} \cdot 7 \mathrm{H}_{2} \mathrm{O}$ 作为催化剂，由氯甲基-甲氧基甲 基-二甲基硅烷 1 制备的格氏试剂能与环丙基甲基酮 (4a)顺利发生反应得到三级醇中间体 $5 \mathrm{a}$, 无需纯化, 进 而以 $\mathrm{Et}_{2} \mathrm{O}$ 作为溶剂, $\mathrm{MgI}_{2} \bullet(\mathrm{OEt})_{n}$ 作为催化剂, 发生 Julia 开环反应，以 $18.6 \%$ 的产率得到 $E / Z$ 构型的混合碘代烯 丙基硅产物 $6 \mathrm{a}, E / Z$ 比例为 $1: 2.1$, 同时得到少量 Peterson 消除产物 7(表 1, Entry 1). 为了进一步提高目标 烯丙基硅产物的产率及其立体选择性，我们考察了不同 溶剂及温度对 Julia 开环反应的影响(表 1).

从表 1 中可以看出, 在 $50{ }^{\circ} \mathrm{C}$ 反应条件下, 在醚类 溶剂中 (乙二醇二甲醚，1,4-二氧六环，THF)，能以更好 的选择性得到 $Z$ 式构型的烯丙基硅化合物(Entries 3 5); 以 $\mathrm{MeCN}$ 作为反应介质，则能显著抑制 Peterson 消 除反应(Entry 6). 分别以 $\mathrm{Et}_{2} \mathrm{O}$ 和 $\mathrm{PhH}$ 作为反应溶剂, 在 不同的温度下反应，发现降低温度，该反应的化学选择 性 $(\mathbf{6 a} / 7 \mathbf{a})$ 和立体选择性 $(E / Z)$ 均未有明显改善(Entries $8 \sim 11)$.

以 $\mathrm{Et}_{2} \mathrm{O}$ 和 $\mathrm{PhH}$ 作为溶剂 ${ }^{[12]}, 50{ }^{\circ} \mathrm{C}$ 反应温度下, 不 同环丙基酮衍生的 $\beta$-差基硅的 Julia 开环反应合成带有 不同取代基的烯丙基硅化合物的情况如表 2 所示. 在实 验条件下，原位合成的带有正己基(Entries 1,2)、高香叶 基(Entries 3,4)和苯基(Entries 5,6)的环丙基醇 5 均能选 择性地发生 Julia 开环反应，得到高碘代烯丙基硅化合 
表 1 溶剂和温度对反应的影响 ${ }^{a}$

Table 1 Effect of solvent and temperature on the reaction
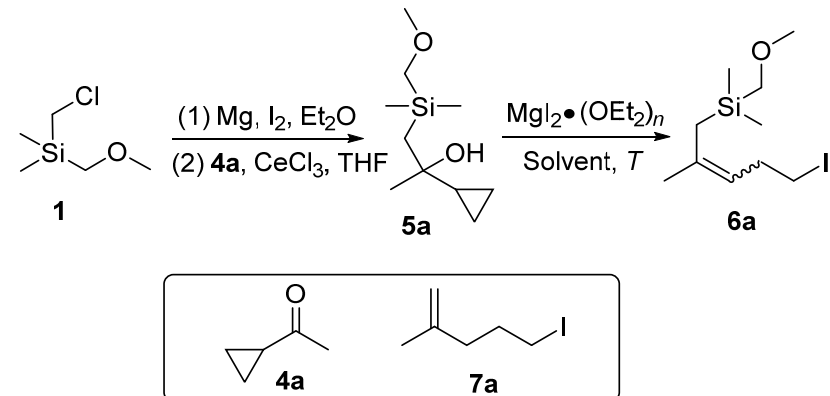

\begin{tabular}{clcccc}
\hline Entry & Solvent & $T /{ }^{\circ} \mathrm{C}$ & Yield $^{b} \%$ & $E: Z^{c}$ & $\mathbf{6 a}: \mathbf{7 a}^{c}$ \\
\hline 1 & $\mathrm{Et}_{2} \mathrm{O}$ & 50 & 18.6 & $1: 2.1$ & $3.4: 1$ \\
2 & $\mathrm{PhH}$ & 50 & 43.3 & $1: 1.0$ & $2.5: 1$ \\
3 & $\mathrm{DME}$ & 50 & 24.0 & $1: 4.5$ & $4.1: 1$ \\
4 & $1,4-$ Dioxane & 50 & 36.7 & $1: 3.4$ & $3.3: 1$ \\
5 & $\mathrm{THF}$ & 50 & 19.5 & $1: 2.7$ & $3.6: 1$ \\
6 & $\mathrm{MeCN}$ & 50 & 41.2 & $1: 1.5$ & $7.1: 1$ \\
7 & $\mathrm{PhMe}$ & 50 & 39.2 & $1: 1.5$ & $1.7: 1$ \\
8 & $\mathrm{Et}_{2} \mathrm{O}$ & 30 & 53.0 & $1: 1.6$ & $1.5: 1$ \\
9 & $\mathrm{Et} 2 \mathrm{O}$ & 0 & 47.8 & $1: 1.5$ & $2.9: 1$ \\
10 & $\mathrm{PhH}_{11}$ & 30 & 41.2 & $1: 1.1$ & $2.3: 1$ \\
11 & $\mathrm{PhH}$ & 0 & 34.1 & $1: 1.8$ & $3.5: 1$ \\
\hline
\end{tabular}

${ }^{a}$ Reaction conditions: tertiary alcohol 5a prepared from the reaction of $\mathbf{1}$ (5.0 $\mathrm{mmol}), 4 \mathrm{a}(3.4 \mathrm{mmol})$ under the presence of $\mathrm{Mg}(5.0 \mathrm{mmol})$ and $\mathrm{CeCl}_{3}(5.0$ $\mathrm{mmol}), \mathrm{MgI}_{2} \cdot(\mathrm{OEt})_{n}(1.0 \mathrm{mmol})$ in dry solvent $(20.0 \mathrm{~mL}) .{ }^{b}$ Isolated yields of 6a based on the loading of $\mathbf{4 a} .{ }^{c}$ Determined by ${ }^{1} \mathrm{H}$ NMR.

物，且与甲基取代的底物相比，带有较大取代基的烯丙 基硅化合物产率得以显著提高. 值得注意的是, 与烷基 取代的三级醇底物 $(\mathbf{5 a} \sim \mathbf{5 c})$ 相比，苯基环丙基酮衍生的 三级醇 5d 发生 Julia 开环反应具有更好的立体选择性, 以 $7: 1$ 的比例得到 $\mathrm{Z}$ 式构型为主的烯丙基硅化合物. 但硅原子上烷氧基的引入, 并没有明显改善高香叶基取 代的烯丙基硅化合物合成的立体选择性 ${ }^{[7 a]}$ (Entries 3,4). 由此可见，利用环丙基甲醇化合物的 Julia 开环反应合 成烯丙基硅化合物的立体选择性与底物结构密切相关.

由于 $E 、 Z$ 构型的烯丙基硅产物极性相似，通过柱 层析无法实现其分离. 构型的确定是通过对比两者 ${ }^{1} \mathrm{H}$ NMR 谱图中形成的双键氢化学位移的相对大小, 并与 已知类似化合物的谱图对照, 结合 1D NOE 谱图确定的. 参照已知类似化合物的谱图数据, 对于高碘代烯丙基硅 化合物，碘代乙基与硅甲基位于双键同侧( $Z$ 式构型)的 异构体中双键氢处于更低场 ${ }^{[8 b]}$ (图 1), 通过对比双键氢 积分比例，从而确定该反应产物的 $E / Z$ 比例.

\section{2 结论}

利用带有烷氧甲基硅基的环丙基甲醇化合物的<smiles>CC(=O)OC[C@@]1(C)O[C@@H]1CC/C=C(\C)CC/C(=C/CCI)CSc1ccccc1</smiles>

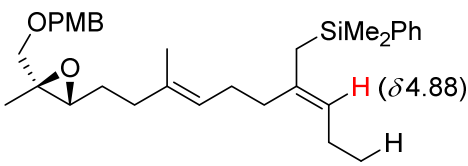

图 1 已知化合物的烯烃氢化学位移

Figure 1 Chemical shift of the vinyl-H in silimar allylsilanes 表 2 带有不同取代基的烯丙基硅的合成 ${ }^{a}$

Table 2 Synthesis of allylsilanes with different substituents
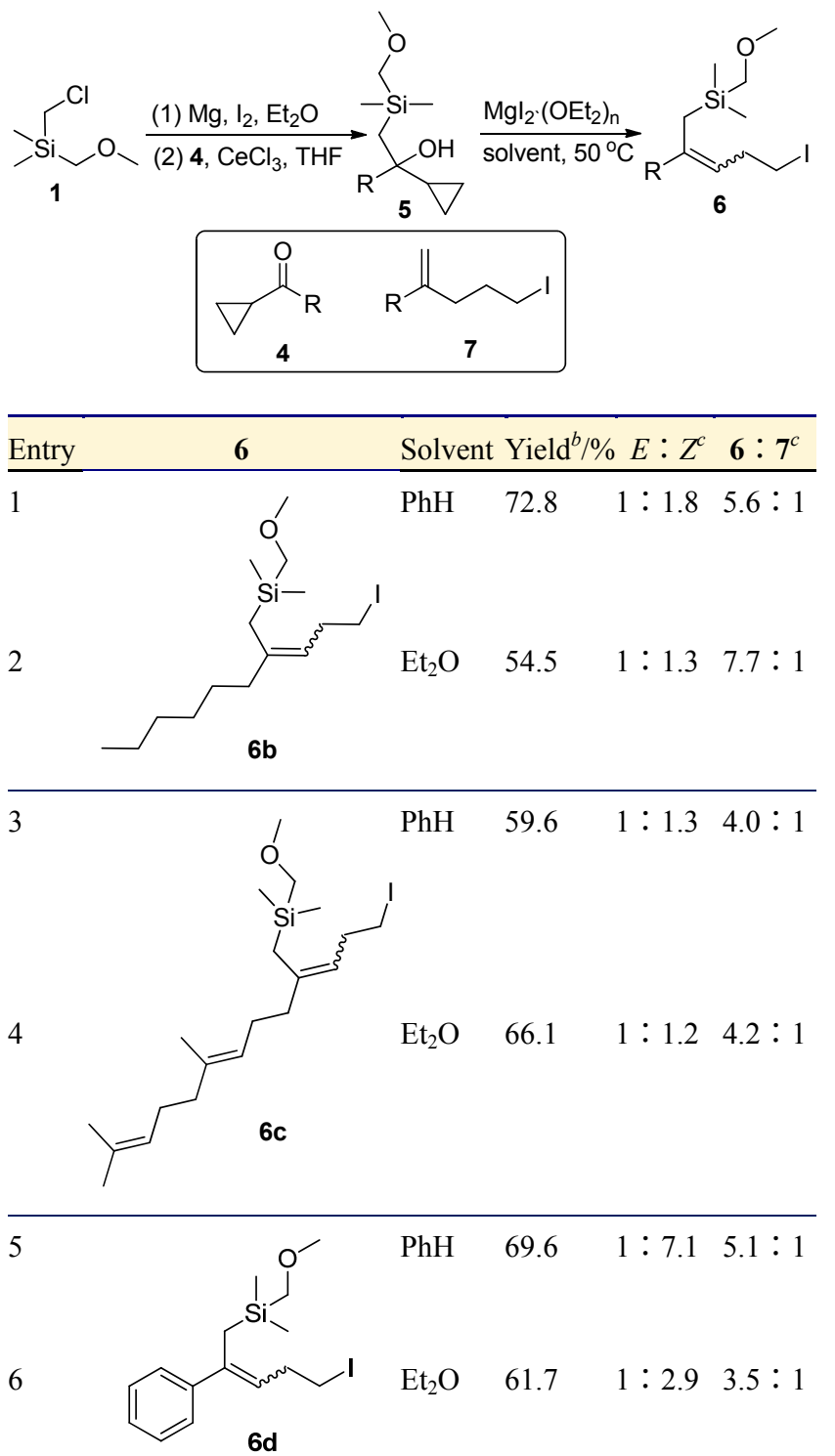

${ }^{a}$ Reaction conditions: tertiary alcohol prepared from the reaction of $1(5.0$
$\mathrm{mmol}), \mathbf{4}(3.35 \mathrm{mmol})$ under the presence of $\mathrm{Mg}(5.0 \mathrm{mmol})$ and $\mathrm{CeCl}_{3}(5.0$
$\mathrm{mmol}), \mathrm{MgI}_{2} \bullet(\mathrm{OEt})_{n}(1.0 \mathrm{mmol})$ in dry solvent $(20.0 \mathrm{~mL})$ at $50{ }^{\circ} \mathrm{C} .{ }^{b}$ Isolated
yields of $\mathbf{6}$ based on the loading of $4 .{ }^{c}$ Determined by ${ }^{1} \mathrm{H}$ NMR.

Julia 烯烃化反应，成功制备了一系列新型烯丙基硅化合 
物. 研究表明, 以 $\mathrm{MgI}_{2} \bullet(\mathrm{OEt})_{n}$ 作为催化剂, 烷基和芳基 取代的环丙基醇底物均能得到 $Z$ 式构型为主的产物. 反 应的立体选择性与底物结构有关, 芳基取代的底物能以 更好的选择性得到 $Z$ 式构型烯丙基硅. 所得到的该类烯 丙基硅化合物同时存在卤原子、 $\mathrm{C}-\mathrm{C}$ 双键和硅原子反 应位点, 是一种潜在的重要有机合成中间体.

\section{3 实验部分}

\section{1 仪器与试剂}

核磁共振氢谱和碳谱采用美国安捷伦 Aglilent DD2 核磁共振仪测定, $\mathrm{CDCl}_{3}$ 为溶剂, TMS 为内标; 高分辨 质谱采用 Acquity SQD 液相色谱-串联质谱联用仪测定. 所用化学试剂和溶剂均为商业可得, 未经特殊说明, 溶 剂均按照标准操作干燥处理后使用, 试剂均是购买后直 接使用. 使用薄层色谱(TLC, GF254 硅胶板)技术监测反 应进程, 显色剂采用磷钿酸、香草醛、单质碘和高锰酸 钾溶液等. 原料 $4 \mathrm{a}$ 和 $4 \mathrm{~d}$ 可商业购买得到, $\mathbf{4} \mathrm{b}^{[12]} 、 \mathbf{4} \mathrm{c}^{[9]}$ 可参照已有文献方法得到.

\section{2 实验方法}

\section{2 .1 氯甲基-甲氧基甲基-二甲基硅烷(1)的合成}

干燥的 $150 \mathrm{~mL}$ 三口瓶中加入新鲜打磨并剪碎的 $\mathrm{Mg}$ 条(200.0 mmol, $4.8 \mathrm{~g}, 1.0$ equiv.)和 $\mathrm{HgCl}_{2}(6.0 \mathrm{mmol}$, $1.6 \mathrm{~g}, 0.03$ equiv.), 置换气体后加入 THF 溶液 $(20 \mathrm{~mL})$. 反应混合液置于 $-20{ }^{\circ} \mathrm{C}$ 低温浴槽中冷却后, 滴加几滴 氯甲基甲基醚( $\mathrm{MOMCl}, 2$ )的干燥 $\mathrm{THF}$ 溶液 $(40 \mathrm{~mL}$ )引发 格氏反应, 随后将反应液冷却至 $-25{ }^{\circ} \mathrm{C}$, 滴加剩下的 MOMCl 溶液(15.2 mL, $16.1 \mathrm{~g}, 200 \mathrm{mmol}, 1.0$ equiv.), 滴 加完毕后, 反应混合液在该温度下继续摚拌反应 $2 \mathrm{~h}$ 得 到格氏试剂 3, 直接用于下一步反应.

将上述制备得到的格氏试剂 3 溶液降温至 $-30{ }^{\circ} \mathrm{C}$, $\mathrm{N}_{2}$ 保护下滴加入氯甲基二甲基氯硅烷 $(15.8 \mathrm{~mL}, 17.2 \mathrm{~g}$, $120 \mathrm{mmol}, 0.6$ equiv.)的干燥 THF 溶液 $(40 \mathrm{~mL})$, 滴加完 毕后, 继续搅拌反应过夜. 薄层色谱(TLC)监测反应完 毕后, 加入甲醇 $(20 \mathrm{~mL})$ 淬灭反应, 乙酸乙酯萃取, 合并 有机相后依次用水和饱和食盐水洗涤, 无水 $\mathrm{Na}_{2} \mathrm{SO}_{4}$ 干 燥, 旋蒸除去溶剂后, 减压蒸馏 $(0.09 \mathrm{MPa})$, 收集 65 $67{ }^{\circ} \mathrm{C}$ 馏分, 得到 $8.4 \mathrm{~g}$ 无色液体 1 , 产率为 $46.0 \%$. ${ }^{1} \mathrm{H}$ NMR $\left(\mathrm{CDCl}_{3}, 400 \mathrm{MHz}\right) \delta$ : 3.34 (s, 3H), 3.20 (s, 2H), 2.84 (s, 2H), 0.15 (s, 6H); ${ }^{13} \mathrm{C}$ NMR $\left(\mathrm{CDCl}_{3}, 100 \mathrm{MHz}\right) \delta: 64.3$, 63.4, 28.7, -6.1; IR (KBr) v: 3850, 3744, 1518, 1244, $456,420 \mathrm{~cm}^{-1}$.

\subsection{2 高碘代烯丙基硅 $6 \mathbf{a} \sim 6 \mathbf{d}$ 的合成}

$\mathrm{N}_{2}$ 保护下 $25 \mathrm{~mL}$ 干燥三口瓶中加入新鲜打磨的镁 屑 $(5.0 \mathrm{mmol}, 120.2 \mathrm{mg})$ 、一粒碘和干燥 $\mathrm{Et}_{2} \mathrm{O}(2.0 \mathrm{~mL})$,
室温下滴加几滴氯甲基甲氧基甲基二甲基硅烷 $(\mathbf{1}$, 溶解 于 $3.0 \mathrm{~mL}$ 干燥 $\mathrm{Et}_{2} \mathrm{O}$ 中), 加热引发格氏反应(碘颜色裉 去, 呈白色浑浊), 然后缓慢滴加剩下的 1 (5.0 mmol, $763.5 \mathrm{mg}$ )的 $\mathrm{Et}_{2} \mathrm{O}$ 溶液, 继续摚拌反应直至固体镁屑完 全消失, 直接用于下一步反应.

干燥的 $50 \mathrm{~mL}$ 三口瓶中加入无水 $\mathrm{CeCl}_{3}(5.0 \mathrm{mmol}$, $1233.0 \mathrm{mg}$ ), 真空条件下加热至 $170{ }^{\circ} \mathrm{C}$ 剧烈搅拌 $2 \mathrm{~h}$ ( $\mathrm{CeCl}_{3}$ 呈类似液体流动的白色固体粉末), 体系中充入 $\mathrm{N}_{2}$, 并冷却至室温后, 加入干燥 THF $(8.0 \mathrm{~mL})$, 剧烈搅 拌 $2 \mathrm{~h}$ 形成 $\mathrm{CeCl}_{3}$ 的均匀悬浊液(保证无结块), 而后加入 环丙基甲基酮 $(4,3.4 \mathrm{mmol}$ )的干燥 THF 溶液 $(6.0 \mathrm{~mL}$ ), 室温下摚拌反应 $2 \mathrm{~h}$ 后, 反应混合液冷却至 $0{ }^{\circ} \mathrm{C}$, 加入 格氏试剂 3. 滴加完毕后, 反应体系缓慢升至室温, TLC 监测反应直至 4 完全消失(约 $30 \mathrm{~min}$ ), 加入饱和 $\mathrm{NaHCO}_{3}$ 溶液 $(3.0 \mathrm{~mL})$ 淬灭反应，乙酸乙酯萃取，合并有 机相, 饱和食盐水洗涤, 无水 $\mathrm{MgSO}_{4}$ 干燥, 浓缩, 所得 粗产物 5 直接用于下一步反应.

$\mathrm{N}_{2}$ 保护下, 将上述制备得到的三级醇 $\mathbf{5}$ 粗产物溶解 于干燥溶剂 $(20.0 \mathrm{~mL})$ 中, $50{ }^{\circ} \mathrm{C}$ 下加入 $\mathrm{MgI}_{2} \cdot(\mathrm{OEt})_{n}$ [0.0625 mol/L in $\mathrm{Et}_{2} \mathrm{O} /$ Benzene $\left.(V / V=1 / 1), 16.0 \mathrm{~mL}\right]$ 溶 液, 滴加完毕后, 继续搅拌反应至 TLC 监测原料完全消 失. 饱和 $\mathrm{NaHCO}_{3}$ 溶液 $(6.0 \mathrm{~mL})$ 淬灭反应, 乙酸乙酯萃 取, 合并有机相, 依次用饱和 $\mathrm{Na}_{2} \mathrm{~S}_{2} \mathrm{O}_{3}$ 溶液、 $\mathrm{H}_{2} \mathrm{O}$ 和饱 和食盐水洗涤, 无水 $\mathrm{MgSO}_{4}$ 干燥, 浓缩, 柱层析 $[V$ (石油 醚) $: V($ 乙酸乙酯 $)=100 ： 1$ ] 得到产物 $\mathbf{6}$.

\section{2 .3 化合物的表征}

2-环丙基-1-[(甲氧基甲基)二甲基硅基]辛-2-醇(5b): 无色油状物. ${ }^{1} \mathrm{H}$ NMR $\left(\mathrm{CDCl}_{3}, 400 \mathrm{MHz}\right) \delta: 3.37$ (s, 3H), $3.16(\mathrm{~s}, 2 \mathrm{H}), 2.17(\mathrm{~s}, 1 \mathrm{H}), 1.49 \sim 1.54(\mathrm{~m}, 2 \mathrm{H}), 1.29 \sim 1.43$ $(\mathrm{m}, 8 \mathrm{H}), 1.08(\mathrm{~s}, 2 \mathrm{H}), 0.82 \sim 0.89(\mathrm{~m}, 4 \mathrm{H}), 0.39 \sim 0.41(\mathrm{~m}$, $2 \mathrm{H}), 0.29 \sim 0.32(\mathrm{~m}, 2 \mathrm{H}), 0.10(\mathrm{~s}, 3 \mathrm{H}), 0.07(\mathrm{~s}, 3 \mathrm{H}) ;{ }^{13} \mathrm{C}$ NMR (CDCl $3,100 \mathrm{MHz}) \delta: 71.7,67.4,63.3,44.6,32.0$, $30.0,28.7,24.4,22.7,21.7,14.1,1.2,1.0,-2.5$, -2.6 ; IR (KBr) v: 3360, 2851, 2379, 1657, $1633 \mathrm{~cm}^{-1}$; HRMS calcd for $\mathrm{C}_{15} \mathrm{H}_{32} \mathrm{NaO}_{2} \mathrm{Si}[\mathrm{M}+\mathrm{Na}]^{+}$295.2064, found 259.2059 .

$(E, E)$-2-环丙基-1-[(甲氧基甲基)二甲基硅基]-6,10二甲基十一-5,9-二烯-2-醇(5c): 无色油状物. ${ }^{1} \mathrm{H}$ NMR $\left(\mathrm{CDCl}_{3}, 400 \mathrm{MHz}\right) \delta: 5.14(\mathrm{t}, J=6.8 \mathrm{~Hz}, 1 \mathrm{H}), 5.09(\mathrm{t}, J=$ $6.8 \mathrm{~Hz}, 1 \mathrm{H}), 3.37$ (s, 3H), $3.18(\mathrm{br}, 1 \mathrm{H}), 3.16(\mathrm{~s}, 2 \mathrm{H})$, $2.04 \sim 2.18(\mathrm{~m}, 4 \mathrm{H}), 1.96 \sim 2.00(\mathrm{~m}, 2 \mathrm{H}), 1.68(\mathrm{~s}, 3 \mathrm{H})$, $1.52 \sim 1.62(\mathrm{~m}, 8 \mathrm{H}), 1.11(\mathrm{~s}, 2 \mathrm{H}), 0.81 \sim 0.88(\mathrm{~m}, 1 \mathrm{H})$, $0.38 \sim 0.42(\mathrm{~m}, 2 \mathrm{H}), 0.28 \sim 0.36(\mathrm{~m}, 2 \mathrm{H}), 0.10(\mathrm{~s}, 3 \mathrm{H})$, 0.08 (s, 3H); ${ }^{13} \mathrm{C} \mathrm{NMR}\left(\mathrm{CDCl}_{3}, 100 \mathrm{MHz}\right) \delta: 134.7,131.2$, 
$124.8,124.3,71.6,67.3,63.2,44.4,39.5,28.8,26.7,25.6$, 23.0, 21.6, 17.6, 15.9, 1.2, 1.1, - 2.5, - 2.6; IR (KBr) v: 3447, 3084, 2964, 2926, 1445, 1250, $1097 \mathrm{~cm}^{-1}$; HRMS calcd for $\mathrm{C}_{20} \mathrm{H}_{38} \mathrm{NaO}_{2} \mathrm{Si}[\mathrm{M}+\mathrm{Na}]^{+}$361.2539, found 361.2530 .

1-环丙基-2-[(甲氧基甲基)二甲基硅基]苯乙醇 $(\mathbf{5 d})$ : 无色油状物. ${ }^{1} \mathrm{H} \mathrm{NMR}\left(\mathrm{CDCl}_{3}, 400 \mathrm{MHz}\right) \delta: 7.45 \sim 7.48$ $(\mathrm{m}, 2 \mathrm{H}), 7.29 \sim 7.32(\mathrm{~m}, 2 \mathrm{H}), 7.18 \sim 7.22(\mathrm{~m}, 1 \mathrm{H}), 4.20(\mathrm{~s}$, $1 \mathrm{H}), 3.33(\mathrm{~s}, 3 \mathrm{H}), 3.02(\mathrm{~d}, J=12.4 \mathrm{~Hz}, 1 \mathrm{H}), 2.88(\mathrm{~d}, J=$ $12.8 \mathrm{~Hz}, 1 \mathrm{H}), 1.58$ (d. $J=14.8 \mathrm{~Hz}, 1 \mathrm{H}), 1.47$ (d, $J=14.8$ $\mathrm{Hz}, 1 \mathrm{H}), 1.20 \sim 1.27(\mathrm{~m}, 1 \mathrm{H}), 0.32 \sim 0.53(\mathrm{~m}, 3 \mathrm{H}), 0.23 \sim$ $0.29(\mathrm{~m}, 1 \mathrm{H}), 0.01(\mathrm{~s}, 3 \mathrm{H}),-0.39(\mathrm{~s}, 3 \mathrm{H}) ;{ }^{13} \mathrm{C} \mathrm{NMR}$ $\left(\mathrm{CDCl}_{3}, 100 \mathrm{MHz}\right) \delta: 149.2,127.7,126.1,125.1,73.6$, 66.9, 63.2, 30.8, 25.6, 2.1, 1.4, - 2.7, -3.6; IR (KBr) v: $3405,2815,1446,1250,1224 \mathrm{~cm}^{-1}$. HRMS calcd for $\mathrm{C}_{15} \mathrm{H}_{24} \mathrm{NaO}_{2} \mathrm{Si}[\mathrm{M}+\mathrm{Na}]^{+}$287.1443, found 287.1453.

( $E / Z$ )-(5-碘-2-甲基-戊-2-烯-1-基)(甲氧基甲基)二甲 基硅烷 $[(E / Z)-6 \mathbf{a}]$ : 无色油状物; ${ }^{1} \mathrm{H}$ NMR $\left(\mathrm{CDCl}_{3}, 400\right.$ $\mathrm{MHz}) \delta: 4.98[\mathrm{t}, J=4.8 \mathrm{~Hz}, 0.5 \mathrm{H},=\mathrm{C}-\mathrm{H}(Z)], 4.94[\mathrm{t}$, $J=4.8 \mathrm{~Hz}, 0.5 \mathrm{H},=\mathrm{C}-\mathrm{H}(E)], 3.35(\mathrm{~s}, 1.5 \mathrm{H}), 3.34(\mathrm{~s}$, $1.5 \mathrm{H}), 3.09 \sim 3.11(\mathrm{~m}, 4 \mathrm{H}), 2.58(\mathrm{q}, J=4.8 \mathrm{~Hz}, 1 \mathrm{H}), 2.52$ $(\mathrm{q}, J=4.8 \mathrm{~Hz}, 1 \mathrm{H}), 1.68(\mathrm{~s}, 1.5 \mathrm{H}), 1.61(\mathrm{~s}, 1.5 \mathrm{H}), 1.58(\mathrm{~s}$, $1 \mathrm{H}), 1.56(\mathrm{~s}, 1 \mathrm{H}), 0.07(\mathrm{~s}, 3 \mathrm{H}), 0.06(\mathrm{~s}, 3 \mathrm{H}) ;{ }^{13} \mathrm{C} \mathrm{NMR}$ $\left(\mathrm{CDCl}_{3}, 100 \mathrm{MHz}\right) \delta: 135.6,135.5,121.3,121.1,66.5$, 66.3, 63.4, 63.4, 32.8, 32.7, 26.9, 26.1, 20.6, 18.9, 6.6, 6.0, -4.0, -4.4; IR (KBr) v: 3842, 3459, 2923, 1638, 1259, $1044,800,548,476 \mathrm{~cm}^{-1}$; HRMS calcd for $\mathrm{C}_{10} \mathrm{H}_{21} \mathrm{INaOSi}$ $[\mathrm{M}+\mathrm{Na}]^{+}$335.0304, found 335.0298.

$(E / Z)$-(2-(3-碘代亚丙基)辛基)(甲氧基甲基)二甲基 硅烷 $[(E / Z)-\mathbf{6 b}]$ : 无色油状物; ${ }^{1} \mathrm{H}$ NMR $\left(\mathrm{CDCl}_{3}, 400\right.$ $\mathrm{MHz}) \delta: 4.96[\mathrm{t}, J=4.8 \mathrm{~Hz}, 0.5 \mathrm{H},=\mathrm{C}-\mathrm{H}(Z)], 4.91(\mathrm{t}$, $J=4.8 \mathrm{~Hz}, 0.5 \mathrm{H},=\mathrm{C}-\mathrm{H}(E)), 3.35(\mathrm{~s}, 1.5 \mathrm{H}), 3.34(\mathrm{~s}$, $1.5 \mathrm{H}), 3.07 \sim 3.11(\mathrm{~m}, 4 \mathrm{H}), 2.51 \sim 2.59(\mathrm{~m}, 2 \mathrm{H}), 1.90 \sim$ $1.95(\mathrm{~m}, 2 \mathrm{H}), 1.57(\mathrm{~s}, 1 \mathrm{H}), 1.54(\mathrm{~s}, 1 \mathrm{H}), 1.26 \sim 1.30(\mathrm{~m}$, $8 \mathrm{H}), 0.87 \sim 0.90(\mathrm{~m}, 3 \mathrm{H}), 0.05(\mathrm{~s}, 6 \mathrm{H}) ;{ }^{13} \mathrm{C} \mathrm{NMR}\left(\mathrm{CDCl}_{3}\right.$, $100 \mathrm{MHz}) \delta$ : 136.1, 126.7, 69.5, 62.1, 36.8, 33.5, 31.3, 29.2, 27.5, 23.0, 14.1, 5.6, 4.0, - 3.7; IR (KBr) v: 3728, 3703, 2921, 1654, 1521, $1167 \mathrm{~cm}^{-1}$. HRMS calcd for $\mathrm{C}_{15} \mathrm{H}_{32} \mathrm{IOSi}[\mathrm{M}+\mathrm{H}]^{+}$383.1267, found 383.1274.

(Z)-[2-(3-碘代亚丙基)-6,10-二甲基-5,9-十二二烯-1基](甲氧基甲基)二甲基硅烷 $[(Z)-6 \mathbf{c}]$ : 无色油状物. ${ }^{1} \mathrm{H}$ NMR $\left(\mathrm{CDCl}_{3}, 400 \mathrm{MHz}\right) \delta: 5.08 \sim 5.13(\mathrm{~m}, 2 \mathrm{H}), 4.99(\mathrm{t}$, $J=6.8 \mathrm{~Hz}, 1 \mathrm{H}), 3.34(\mathrm{~s}, 3 \mathrm{H}), 3.08 \sim 3.11(\mathrm{~m}, 4 \mathrm{H}), 2.50 \sim$ $2.56(\mathrm{~m}, 2 \mathrm{H}), 2.04 \sim 2.14(\mathrm{~m}, 4 \mathrm{H}), 1.92 \sim 1.99(\mathrm{~m}, 4 \mathrm{H})$, $1.68(\mathrm{~s}, 3 \mathrm{H}), 1.59 \sim 1.60(\mathrm{~m}, 8 \mathrm{H}), 0.06(\mathrm{~s}, 6 \mathrm{H}) ;{ }^{13} \mathrm{C} \mathrm{NMR}$
$\left(\mathrm{CDCl}_{3}, 100 \mathrm{MHz}\right) \delta: 139.2,135.1,131.2,124.3,124.0$, 120.6, 66.5, 63.4, 39.7, 39.0, 32.7, 26.8, 26.6, 25.7, 18.7, 17.7, 16.1, 6.0, - 4.0; IR (KBr) v: 3363, 3205, 2853, $2379,1636,837,736 \mathrm{~cm}^{-1}$. HRMS calcd for $\mathrm{C}_{20} \mathrm{H}_{37} \mathrm{INaO}-$ $\mathrm{Si}[\mathrm{M}+\mathrm{Na}]^{+}$471.1556, found 471.1555 .

(Z)-(5-碘-2-苯基-戊-2-烯-1-基)(甲氧基甲基)二甲基 硅烷 $[(Z)-6 d]$ : 无色油状物; ${ }^{1} \mathrm{H} \mathrm{NMR}\left(\mathrm{CDCl}_{3}, 400 \mathrm{MHz}\right)$ $\delta: 7.23 \sim 7.36(\mathrm{~m}, 5 \mathrm{H}), 5.46(\mathrm{t}, J=7.0 \mathrm{~Hz}, 1 \mathrm{H}), 3.20 \sim 3.23$ $(\mathrm{m}, 5 \mathrm{H}), 2.88(\mathrm{~s}, 2 \mathrm{H}), 2.72 \sim 2.77(\mathrm{~m}, 2 \mathrm{H}), 2.06(\mathrm{~s}, 2 \mathrm{H})$, $-0.11(\mathrm{~s}, 6 \mathrm{H}) ;{ }^{13} \mathrm{C} \mathrm{NMR}\left(\mathrm{CDCl}_{3}, 100 \mathrm{MHz}\right) \delta$ : 144.0, $139.9,128.1,127.0,126.5,124.4,66.1,63.2,33.2,18.4$, 5.3, -4.3; IR (KBr) v: 3455, 2965, 2928, 1639, 1072 , $977,804 \mathrm{~cm}^{-1}$. HRMS calcd for $\mathrm{C}_{15} \mathrm{H}_{24} \mathrm{INaOSi}[\mathrm{M}+\mathrm{Na}]^{+}$ 397.0461 , found 397.0468

辅助材料(Supporting Information) 化合物 $\mathbf{1 ,}, \mathbf{5 b} \sim \mathbf{5 d}$ 和 6a $\sim 6 \mathbf{d}$ 的 ${ }^{1} \mathrm{H} N \mathrm{NMR}$ 和 ${ }^{13} \mathrm{C} \mathrm{NMR}$ 谱图. 这些材料可以 免费从本刊网站(http://sioc-journal.cn/)上下载.

\section{References}

[1] Sommer, L. H.; Tyler, L. J.; Whitmore, F. C. J. Am. Chem. Soc. 1948, 70, 2872.

[2] For recent reviews, see: (a) Ramachandran, P. V.; Nicponski, D. R.; Gagare, P. D. In Comprehensive Organic Synthesis II, Vol. 2, Eds.: Knochel, P.; Molander, G. A., Elsevier, Amsterdam, 2014, p. 72.

(b) Díez-Poza, C.; Barbero, A. Eur. J. Org. Chem. 2017, 2017, 4651.

(c) Schmidt, A. W.; Knölker, H.-J. Synlett 2010, 2207.

(d) Chabaud, L.; James, P.; Landais, Y. Eur. J. Org. Chem. 2004, 3173 .

(e) Barbero, A.; Pulido, F. J. Acc. Chem. Res. 2004, 37, 817.

(f) Méndez, M.; Echavarren, A. M. Eur. J. Org. Chem. 2002, 15.

(g) Fleming, I.; Barbero, A.; Walter, D. Chem. Rev. 1997, 97, 2063.

[3] Langkopf, E.; Schinzer, D. Chem. Rev. 1995, 95, 1375.

[4] For reviews on the synthesis of allylsilanes see 2a, and also see: (a) Sarkar, T. K. Synthesis 1990, 969

(b) Sarkar, T. K. Synthesis 1990, 1101.

(c) Suginome, M.; Ito, Y. J. Organomet. Chem. 2003, 680, 43. (d) Barbero, A.; Pulido, F. J. Synthesis 2004, 779.

[5] Han, J. W.; Hayashi, T. Tetrahedron: Asymmetry 2010, 21, 2193 and references therein

[6] (a) Chan, T. H.; Wang, D. Chem. Rev. 1992, 92, 995.

(b) Masse, C. E.; Panek, J. S. Chem. Rev. 1995, 95, 1293.

For selected examples on transformations of chiral allylsilanes, see: (c) Hayashi, T.; Konishi, M.; Kumada, M. J. Am. Chem. Soc. 1982, 104, 4963

(d) Wang, D. Chin. J. Org. Chem. 2001, 21, 1090 (in Chinese). (王东, 有机化学, 2001, 21, 1090.)

(e) Wu, J.; Pu, Y.; Panek, J. S. J. Am. Chem. Soc. 2012, 134, 18440.

[7] (a) Li, W.-D. Z.; Yang, J.-H. Org. Lett. 2004, 6, 1849.

(b) Yang, J. H.; Li, W. D. Z. Chin. Chem. Lett. 2005, 16, 433.

[8] (a) Shen, S.-J.; Li, W.-D. Z. J. Org. Chem. 2013, 78, 7112. 
(b) Gao, H.-T.; Wang, B.-L.; Li, W.-D. Z. Tetrahedron 2014, 70, 9436.

[9] Li, W.-D. Z.; Peng, Y. Org. Lett. 2005, 7, 3069.

[10] Lawrence, N. J. Science of Synthesis (Houben-Weyl), Georg Thieme Verlag, Stuttgart, Germany, 2002, pp. 579 594 .

[11] Methoxymethyl magnesium chloride is known to be obtained at low temperature but decomposes above $-20{ }^{\circ} \mathrm{C}$ to release ethylene gas, see: (a) Normant, H.; Castro, B. C. R. Acad. Sci. 1963, 257, 2115.

(b) Castro, B. Bull. Soc. Chim. Fr. 1967, 1533.

[12] Scheiper, B.; Bonnekessel, M.; Krause, H.; Fürstner, A. J. Org. Chem. 2004, 69, 3943.

(Lu, Y.) 